\title{
Practice guideline recommendations summary: Disease-modifying therapies for adults with multiple sclerosis
}

\author{
Report of the Guideline Development, Dissemination, and Implementation \\ Subcommittee of the American Academy of Neurology
}

\begin{abstract}
Alexander Rae-Grant, MD, Gregory S. Day, MD, MSc, Ruth Ann Marrie, MD, PhD, Alejandro Rabinstein, MD, Bruce A.C. Cree, MD, PhD, MAS, Gary S. Gronseth, MD, Michael Haboubi, DO, June Halper, MSN, APN-C, MSCN, Jonathan P. Hosey, MD, David E. Jones, MD, Robert Lisak, MD, Daniel Pelletier, MD, Sonja Potrebic, MD, PhD, Cynthia Sitcov, Rick Sommers, LMSW, Julie Stachowiak, PhD, Thomas S.D. Getchius, Shannon A. Merillat, MLIS, and Tamara Pringsheim, MD, MSc
\end{abstract}

Neurology ${ }^{\circledR}$ 2018;90:777-788. doi:10.1212/WNL.0000000000005347

Abstract
Objective
To develop recommendations for disease-modifying therapy (DMT) for multiple sclerosis (MS).

\section{Methods}

A multidisciplinary panel developed DMT recommendations, integrating findings from a systematic review; followed an Institute of Medicine-compliant process to ensure transparency and patient engagement; and developed modified Delphi consensus-based recommendations concerning starting, switching, and stopping DMTs pertinent to people with relapsingremitting MS, secondary progressive MS, primary progressive MS, and clinically isolated syndromes of demyelination. Recommendations were supported by structured rationales, integrating evidence from one or more sources: systematic review, related evidence (evidence not from the systematic review), principles of care, and inference from evidence.

\section{Results}

Thirty recommendations were developed: 17 on starting DMTs, including recommendations on who should start them; 10 on switching DMTs if breakthrough disease develops; and 3 on stopping DMTs. Recommendations encompassed patient engagement strategies and individualization of treatment, including adherence monitoring and disease comorbidity assessment. The panel also discussed DMT risks, including counseling about progressive multifocal leukoencephalopathy risk in people with MS using natalizumab, fingolimod, rituximab, ocrelizumab, and dimethyl fumarate; and made suggestions for future research to evaluate relative merits of early treatment with higher potency DMTs vs standard stepped-care protocols, DMT comparative effectiveness, optimal switching strategies, long-term effects of DMT use, definitions of highly active MS, and effects of treatment on patient-specified priority outcomes. This guideline reflects the complexity of decisionmaking for starting, switching, or stopping MS DMTs. The field of MS treatment is rapidly changing; the Academy of Neurology development process includes planning for future updates.

\author{
Correspondence \\ American Academy of \\ Neurology \\ guidelines@aan.com
}

\section{MORE ONLINE}

ค Podcast

Dr. Stacey Clardy interviews

Dr. Alexander Rae-Grant about his paper on diseasemodifying therapies for adults with MS.

NPub.org/p5cuqd

RELATED ARTICLES

\section{Editorial}

Complexity of MS

management in the current treatment era

Page 761

\section{Article}

Comprehensive systematic review summary: Diseasemodifying therapies for adults with multiple sclerosis: Report of the Guideline Development, Dissemination, and Implementation Subcommittee of the American Academy of Neurology

Page 789 AMERICAN ACADEMY OF NEUROLOGY.

\footnotetext{
From the Department of Neurology (A.R.-G.), Cleveland Clinic, OH; Department of Neurology (G.S.D.), Charles F. and Joanne Knight Alzheimer Disease Research Center, Washington University in St. Louis, MO; Department of Community Health Sciences (R.A.M.), Max Rady College of Medicine, Rady Faculty of Health Sciences, University of Manitoba, Winnipeg, Canada; Department of Neurology (A.R.), Mayo Clinic, Rochester, MN; UCSF Weill Institute for Neurosciences, Department of Neurology (B.A.C.C.), University of California, San Francisco; Department of Neurology (G.S.G.), Kansas University Medical Center, Kansas City; Department of Neurology (M.H.), School of Medicine, University of Louisville, KY; Consortium of Multiple Sclerosis Centers (J.H.), Hackensack, NJ; Department of Neuroscience (J.P.H.), St. Luke's University Health Network, Bethlehem, PA; Department of Neurology (D.E.J.), University of Virginia, Charlottesville; Consortium of Multiple Sclerosis Centers (R.L.), Hackensack, NJ; Department of Neurology (R.L.), School of Medicine, Wayne State University, Detroit, MI; Department of Neurology (D.P.), Keck School of Medicine, University of Southern California; Neurology Department (S.P.), Southern California Permanente Medical Group, Kaiser, Los Angeles; National Multiple Sclerosis Society (C.S.), Arlington, VA; National Multiple Sclerosis Society (R.S.), New York, NY; Santa Fe (J.S.), NM; Heart Rhythm Society (T.S.D.G.), Washington, DC; American Academy of Neurology (S.A.M.), Minneapolis, MN; and Department of Clinical Neurosciences, Psychiatry, Pediatrics and Community Health Sciences, Cumming School of Medicine (T.P.), University of Calgary, Alberta, Canada.
}

Go to Neurology.org/N for full disclosures. Funding information and disclosures deemed relevant by the authors, if any, are provided at the end of the article.

Approved by the Guideline Development, Dissemination, and Implementation Subcommittee on October 9, 2017; by the Practice Committee on October 21, 2017; and by the AAN Institute Board of Directors on March 6, 2018 


\section{Glossary}

AAN = American Academy of Neurology; AE = adverse effect; CIS = clinically isolated syndrome; $\mathbf{D M T}=$ disease-modifying therapy; EDSS = Expanded Disability Status Scale; FDA = Food and Drug Administration; IFN- $\beta=$ interferon $-\beta$; JCV = John Cunningham virus; $\mathbf{M S}$ = multiple sclerosis; PML = progressive multifocal leukoencephalopathy; $\mathbf{P P M S}$ = primary progressive multiple sclerosis; $\mathbf{R C T}$ = randomized controlled trial; REMS = risk evaluation and mitigation strategies; RRMS = relapsingremitting multiple sclerosis; SPMS = secondary progressive multiple sclerosis.

This article presents the recommendations and suggestions for future research of an American Academy of Neurology (AAN) practice guideline on the efficacy and safety of diseasemodifying therapies (DMTs) in multiple sclerosis (MS). The complete practice guideline and references el through e49, cited here, are available at links.lww.com/WNL/A458 and links.lww.com/WNL/A377, respectively. A companion article summarizes the systematic review findings and conclusions. This guideline, although not a formal update to the 2002 AAN guideline on DMTs, ${ }^{1}$ replaces that earlier guideline.

In May 2015, the AAN guideline subcommittee convened a multidisciplinary panel to perform a systematic review and develop guideline recommendations regarding DMT for MS. The systematic review was completed in November 2016; its methodology and results are described in a companion systematic review. ${ }^{2}$ The panel developed recommendations, founded on the systematic review, concerning the starting, switching, and stopping of DMTs. This summary article focuses on the recommendations and does not include detailed analysis of the systematic review. Readers are urged to review the fulllength document (links.lww.com/WNL/A458) and accompanying tables (appendix e-5, links.lww.com/WNL/A376) for a fuller assessment of this guideline. ${ }^{1}$

Much more than evidence must be considered when crafting practice recommendations. The evidence-based conclusions from the systematic review form the foundation of the AAN process, ${ }^{3}$ but additional factors influence recommendation structure. Working in teams, the panel developed rationale statements for transparency in documenting the deductive logic justifying each recommendation. A rationale statement precedes each recommendation or recommendation set. Four premise types can support recommendations: evidence-based conclusions from the systematic review, generally accepted principles of care, strong evidence from related conditions, and deductive inferences from other premises. Recommendations must be supported by at least one premise.

When there is sufficient evidence to support an inference for an intervention's use (i.e., the balance of benefits and harms favors the intervention), the development panel assigns 1 of 3

\section{$\oplus$ Supplemental Data}

Practice Guidelines

NPub.org/77nyxt recommendation designations: A, B, or C. Each designation corresponds to a helping verb that denotes the level of recommendation strength. Level A is the strongest recommendation level and is denoted by use of the helping verb must. These recommendations are rare, as they are based on high confidence in the evidence and require both a high magnitude of benefit and low risk. Level B corresponds to the helping verb should. Such recommendations are more common, as the requirements are less stringent but still based on the evidence and benefit-risk profile. Level $\mathrm{C}$, which corresponds to the helping verb may, represents the lowest allowable recommendation level the AAN considers useful within the scope of clinical practice and can accommodate the highest degree of practice variation.

Other factors that need to be transparently and systematically considered when formulating recommendations include the relative value of the benefit compared with the risk, the feasibility of complying with the intervention (e.g., the intervention's availability), the intervention's cost, and the expected variation in patient preferences relative to the intervention's risks, burdens, and benefits.

The panel assigned levels of obligation ( $\mathrm{A}, \mathrm{B}, \mathrm{C}$, or $\mathrm{U})$ to the recommendations, using a modified Delphi process that synthesizes all the preceding factors. The panelists provided their opinions concerning the importance of each factor through an online questionnaire, with statistical analysis of responses. All panelists voted online anonymously and independently on each recommendation in 3 voting rounds that involved precisely defined rules for consensus.

Each recommendation achieved consensus, was revised, or was not carried forward. In some cases, the panel reviewed, revised, and revoted on recommendations on the basis of public commentary and other input during the development process, reflecting the dynamic nature of this process.

For the systematic review, observational data did not drive conclusions, but the panel was not restricted in the recommendation phase from reflecting such data if appropriate in the process. In addition, longer-term safety and outcome data are often unavailable for newer agents, and guidelines cannot capture all the longitudinal changes in practice that may occur on the basis of clinical experience over time.

Suggestions for future research were also developed during the guideline development process. 
This guideline will be reassessed periodically for currency and will be updated in accordance with the most currently available AAN process manual.

\section{Starting DMT: Recommendations}

\section{Starting: Recommendation 1}

\section{Rationale}

Receiving the diagnosis of MS is a stressful life event. ${ }^{4,5}$ People receiving major diagnoses may not recall much of the information given to them at the time. ${ }^{6}$ Providing information about DMT at a follow-up interaction is likely to allow a better understanding of these medications and their risks and benefits.

\section{Statement 1}

Clinicians should counsel people with newly diagnosed MS about specific treatment options with DMT at a dedicated treatment visit (Level B).

\section{Starting: Recommendation 2}

\section{Rationale}

Respecting patient preferences is an important component of care for chronic conditions. Because of the variety of DMTs available, evaluating patient preferences may improve acceptance of and adherence to DMT.

\section{Statement 2a}

Clinicians must ascertain and incorporate/review preferences in terms of safety, route of administration, lifestyle, cost, efficacy, common adverse effects (AEs), and tolerability in the choice of DMT in people with MS being considered for DMT (Level A).

\section{Statement 2b}

Clinicians must engage in an ongoing dialogue regarding treatment decisions throughout the disease course with people with MS (Level A).

\section{Starting: Recommendation 3}

\section{Rationale}

DMTs reduce but do not eliminate MS relapses and MRI activity. Educating people with MS about realistic expectations regarding DMT effects is important. ${ }^{7}$ Clinicians should inform people with MS that they may still need symptomatic treatment in addition to DMT. 8

\section{Statement 3a}

Clinicians should counsel people with MS that DMTs are prescribed to reduce relapses and new MRI lesion activity. DMTs are not prescribed for symptom improvement in people with MS (Level B).

\section{Statement 3b}

Clinicians must counsel people with MS on DMTs to notify the clinicians of new or worsening symptoms (Level A).

\section{Starting: Recommendation 4}

\section{Rationale}

Because DMT use requires commitment to ongoing therapy and an understanding of AEs, readiness to initiate DMT and factors causing reluctance may have an impact on adherence to DMT use.

\section{Statement 4}

Clinicians should evaluate readiness or reluctance to initiate DMT and counsel on its importance in people with MS who are candidates to initiate DMT (Level B).

\section{Starting: Recommendation $\mathbf{5}$}

\section{Rationale}

In people with MS, comorbid disease, such as depression, anxiety, and vascular risk factors, and adverse health behaviors (e.g., physical inactivity, smoking) are associated with worse outcomes. ${ }^{9,10}$ Addressing depression before initiating DMT may improve decision-making and adherence to DMT. Concomitant medications may have important interactions with DMTs. $^{11}$

\section{Statement 5}

Clinicians should counsel about comorbid disease, adverse health behaviors, and potential interactions of the DMT with concomitant medications when people with MS initiate DMTs (Level B).

\section{Starting: Recommendation 6}

\section{Rationale}

Because DMT requires adherence to treatment to provide full efficacy, and because that adherence to treatment may be an issue for people with $\mathrm{MS},{ }^{12,13}$ discussing adherence issues before initiating DMT is part of good clinical practice. Efforts to increase adherence may improve outcomes.

\section{Statement $6 \mathbf{a}$}

Clinicians should evaluate barriers to adherence to DMT in people with MS (Level B).

\section{Statement 6b}

Clinicians should counsel on the importance of adherence to DMT when people with MS initiate DMTs (Level B).

\section{Starting: Recommendation 7}

\section{Rationale}

People presenting with a first demyelinating event who do not meet the 2010 International Criteria for MS are commonly encountered in clinical practice. Multiple prospective observational trials have consistently confirmed that people with a single clinical demyelinating event with 2 or more brain or spinal cord lesions remain at increased risk of a future MS diagnosis, with the highest risk incurred within 5 years of the initial event. ${ }^{14-17}$ Evidence from multiple Class I and II trials confirms that DMTs are associated with a significant delay in 
second clinical relapse or new brain MRI-detected lesions in people with a first demyelinating event who are considered to be at high risk for MS on the basis of brain MRI-detected lesions. There is insufficient evidence concerning the comparative efficacy of specific DMTs for this purpose. Decisions concerning the selection of specific DMTs for people presenting with a first demyelinating event should abide by prescribing principles espoused in other recommendations. Individuals presenting with an incident demyelinating event who have no brain lesions are at low risk of a future MS diagnosis.

\section{Statement 7a}

Clinicians should discuss the benefits and risks of DMTs for people with a single clinical demyelinating event with 2 or more brain lesions that have imaging characteristics consistent with MS (Level B).

\section{Statement 7b}

After discussing the risks and benefits, clinicians should prescribe DMT to people with a single clinical demyelinating event and 2 or more brain lesions characteristic of MS who decide they want this therapy (Level B).

\section{Starting: Recommendation 8}

\section{Rationale}

The benefit of initiating DMT has not been studied in currently untreated people with clinically isolated syndromes (CIS) or relapsing forms of MS who have not had relapses in 2 or more years and do not have active new MRI lesion activity on recent imaging. In such people, it is unknown what the risk of harm is from initiating DMTs, including AEs, major AEs, and burden of taking a long-term medication, relative to the benefit of reducing relapse rate.

\section{Statement 8}

Clinicians may recommend serial imaging at least annually for the first 5 years and close follow-up rather than initiating DMT in people with CIS or relapsing forms of MS who are not on DMT, have not had relapses in the preceding 2 years, and do not have active new MRI lesion activity on recent imaging (Level C).

\section{Starting: Recommendation 9}

\section{Rationale}

Multiple studies of DMTs in people with relapsing forms of MS who have had recent relapses or MRI activity or both have shown benefit of DMT in terms of reducing relapses and reducing MRI activity. This includes people with a single clinical episode who meet 2010 International Criteria for MS. ${ }^{18,19}$

\section{Statement 9}

Clinicians should offer DMTs to people with relapsing forms of MS with recent clinical relapses or MRI activity (Level B).

\section{Starting: Recommendation 10}

\section{Rationale}

Lack of adherence to treatment of chronic diseases is a wide-ranging problem. The result of poor adherence is reduced effectiveness and increased health care costs. ${ }^{20-25}$ Regular interactions and assessments by clinicians facilitate prompt identification and treatment of AEs, increased tolerability of the medication, and safety monitoring. ${ }^{7,25}$ Some DMTs for MS have specific risk evaluation and mitigation strategies (REMS) with recommendations for follow-up frequency. ${ }^{26-29}$

\section{Statement 10a}

Clinicians should monitor for medication adherence, AEs, tolerability, safety, and effectiveness of the therapy in people with MS on DMTs (Level B).

\section{Statement 10b}

Clinicians should follow up either annually or according to medication-specific REMS in people with MS on DMTs (Level B).

\section{Starting: Recommendation 11}

\section{Rationale}

DMTs have potential risks in pregnant women ${ }^{30}$ to varying degrees. Discussing pregnancy with women with MS before initiating DMT is a part of good clinical practice. If women with MS are planning pregnancy soon, DMT use may need to be deferred until after pregnancy. ${ }^{31}$ In addition, because DMTs vary in terms of pregnancy risks, ${ }^{30}$ DMT choice may be influenced by plans for pregnancy.

\section{Statement 11}

Clinicians should monitor the reproductive plans of women with MS and counsel regarding reproductive risks and use of birth control during DMT use in women of childbearing potential who have MS (Level B).

\section{Starting: Recommendation 12}

\section{Rationale}

Chemotherapy, such as cyclophosphamide, may affect male fertility. ${ }^{32}$ With teriflunomide treatment, there may be a risk of teratogenicity from male sperm, which could last for 2 years after treatment cessation if the patient is not treated with chelation therapy. ${ }^{33}$

\section{Statement 12}

Clinicians should counsel men with MS on their reproductive plans regarding treatment implications before initiating treatment with teriflunomide or cyclophosphamide (Level B).

\section{Starting: Recommendation 13}

\section{Rationale}

Post approval of mitoxantrone, new evidence has shown a high risk of cardiomyopathy, ovarian failure, male infertility, 
chromosomal aberrations, and promyelocytic leukemia ${ }^{34-37}$ associated with mitoxantrone use. Other effective medications with lower risk, which were unavailable at the time of US Food and Drug Administration (FDA) approval of mitoxantrone, are now available for treating MS.

\section{Statement 13}

Because of the high frequency of severe AEs, clinicians should not prescribe mitoxantrone to people with MS unless the potential therapeutic benefits greatly outweigh the risks (Level B).

\section{Starting: Recommendation 14}

\section{Rationale}

MS is a heterogeneous disease and is characterized by highly variable degrees of disease activity in the relapsing phase and by varying rates of worsening during the progressive phases. ${ }^{38,39}$ Definitions of highly active MS vary and can include measures of relapsing activity and MRI markers of disease activity, such as numbers of gadolinium-enhanced lesions. ${ }^{40, \mathrm{e} 1}$ Subgroup analyses from phase III pivotal trials of alemtuzumab, fingolimod, and natalizumab showed a reduction in relapses and MRI measures in people with MS with highly active disease. ${ }^{\mathrm{e} 2-\mathrm{e} 4}$ Compared with interferon- $\beta$ $(\mathrm{IFN}-\beta)$ therapy, treatment with these therapies resulted in more favorable outcomes in the subgroup of people with MS with highly active disease. ${ }^{\mathrm{e}-\mathrm{e} 8}$ However, the risks and benefits of each treatment strategy need to be considered on a patientby-patient basis.

\section{Statement 14}

Clinicians should prescribe alemtuzumab, fingolimod, or natalizumab for people with MS with highly active MS (Level B).

\section{Starting: Recommendation 15}

\section{Rationale}

DMTs should be available to all people with relapsing forms of MS. Because of disparities in health care provision in different settings, ${ }^{\mathrm{e} 9}$ there may be situations where approved DMTs are not available to an individual. In these situations, DMTs may be obtained with support from the pharmaceutical industry or from organizations, such as the National Organization of Rare Diseases, county organizations, or government organizations. If such support is unavailable, certain lower cost medications may become a choice for care. Azathioprine has mixed results and evidence for which confidence is low to support efficacy in relapsing forms of MS. Cladribine has evidence of benefit for both the oral and parenteral formulations, but currently only the parenteral formulations are available.

\section{Statement 15a}

Clinicians may direct people with MS who are candidates for DMTs to support programs (Level C).

\section{Statement 15b}

Clinicians may recommend azathioprine or cladribine for people with relapsing forms of MS who do not have access to approved DMTs (Level C).

\section{Starting: Recommendation 16}

\section{Rationale}

People with MS with a positive John Cunningham virus (JCV) antibody test have a higher risk of developing progressive multifocal leukoencephalopathy (PML) while using natalizumab, particularly people with MS who have been treated for more than 2 years or have had prior immunosuppressive treatment. There are now other highly effective treatments that may be used that have not been shown to have a similar PML risk. The PML risk increases with the level of anti-JCV antibody response (index). For example, in those using natalizumab for 25-36 months with no prior use of immunosuppressants, the PML risk is 0.2 per 1,000 in those with an index of 0.9 or less, 0.3 per 1,000 in those with an index of $0.9-1.5$, and 3 per 1,000 in those with an index greater than 1.5. Further data on risk assessment is likely to become available over time to help inform treatment decisions in this area.

\section{Statement 16}

Clinicians may initiate natalizumab treatment in people with MS with positive anti-JCV antibody indexes above 0.9 only when there is a reasonable chance of benefit compared with the low but serious risk of PML (Level C).

\section{Starting: Recommendation 17}

\section{Rationale}

Ocrelizumab is the only DMT shown to alter disease progression in individuals with primary progressive MS (PPMS) who are ambulatory. The randomized controlled trial (RCT) of rituximab in PPMS was promising but inconclusive. ${ }^{\text {e10 }}$ Although RCTs of fingolimod, glatiramer acetate, and IFN$\beta-1 b$ failed to demonstrate an effect on disability progression in individuals with PPMS, significant effects on MRI measures of disease activity were found with all 3 treatments. ${ }^{\text {el1-e13 }}$ Clinical trials have not evaluated the benefits of DMT in individuals with PPMS who are nonambulatory with respect to other clinically relevant domains, including vision, cognition, and upper limb function.

\section{Statement 17}

Clinicians should offer ocrelizumab to people with PPMS who are likely to benefit from this therapy unless there are risks of treatment that outweigh the benefits (Level B).

\section{Switching DMT: Recommendations}

\section{Switching: Recommendation 1}

\section{Rationale}

Ongoing disease activity, measured either by clinical relapses or new MRI-detected lesions (including unequivocally new 
T2 or new gadolinium-enhanced lesions), could lead to physical or cognitive worsening over time. ${ }^{\text {e14-e17 }}$ Now that several DMTs are available and have demonstrated efficacy for the prevention of clinical relapses and new MRI-detected lesions, physicians and people with MS often face the decision of switching from one DMT to another because of a perceived lack of efficacy. Such lack of response to a DMT has been difficult to define, as most people with MS are not free of all disease activity; investigators have considered using the number of clinical attacks or new MRI-detected lesions in the preceding 12 months to define lack of response. ${ }^{\mathrm{e} 15, \mathrm{e} 17} \mathrm{DMTs}$ take a variable amount of time to become clinically active, and new lesion formation may occur after initiation but before the time of full efficacy, confounding interpretation of follow-up MRI scans. ${ }^{\mathrm{e} 5, \mathrm{e} 6, \mathrm{e} 18-\mathrm{e} 21}$ Consequently, many clinicians obtain new baseline MRI 3-6 months after initiating DMTs to monitor from a treated baseline. ${ }^{\text {e22 }}$ The optimal interval for ongoing monitoring is uncertain, as short-term stability as evidenced by clinical and MRI criteria may not consistently predict long-term stability. In addition, because of different mechanisms of activity among the DMTs, monitoring strategies may vary.

\section{Statement 1a}

Clinicians should monitor MRI disease activity from the clinical onset of disease to detect the accumulation of new lesions in order to inform treatment decisions in people with MS using DMTs (Level B).

\section{Statement 1b}

Clinicians should recognize that relapses or new MRIdetected lesions may develop after initiation of a DMT and before the treatment becomes effective in people with MS who are using DMTs (Level B).

\section{Statement 1c}

Clinicians should discuss switching from one DMT to another in people with MS who have been using a DMT long enough for the treatment to take full effect and are adherent to their therapy when they experience 1 or more relapses, 2 or more unequivocally new MRI-detected lesions, or increased disability on examination, over a 1-year period of using a DMT (Level B).

\section{Switching: Recommendation 2}

\section{Rationale}

None of the available DMTs is completely effective against relapses and MRI activity. When a patient shows breakthrough disease activity (continued relapses, MRI activity), trying a medication with a different mechanism or efficacy profile may be beneficial. Although all possible clinical scenarios cannot be answered by drug trials, current evidence supports higher efficacy of alemtuzumab, natalizumab, fingolimod, and ocrelizumab compared with previously approved self-injectable DMTs. Tolerability and likelihood of adherence are other factors that are important in decisions about switching DMTs. Physician judgment and patient preferences are critical in this process.

\section{Statement 2}

Clinicians should evaluate the degree of disease activity, adherence, AE profiles, and mechanism of action of DMTs when switching DMTs in people with MS with breakthrough disease activity during DMT use (Level B).

\section{Switching: Recommendation 3}

\section{Rationale}

Multiple DMTs are available for MS treatment. Switching therapies may be appropriate in people with MS who are experiencing AEs or complications with a DMT. Adherence to injectable DMTs is often incomplete. ${ }^{\text {e23 }}$ Injection fatigue (physical or emotional) or injection-related pain or discomfort may be a common reason for poor adherence.

\section{Statement 3}

Clinicians should discuss a change to noninjectable or less frequently injectable DMTs in people with MS who report intolerable discomfort with the injections or in those who report injection fatigue on injectable DMTs (Level B).

\section{Switching: Recommendation 4}

\section{Rationale}

Adherence to a DMT may also be affected by medication AEs. $^{13,25}$ All DMTs have common AEs that may affect adherence (table e-2, links.lww.com/WNL/A376).

\section{Statement $\mathbf{4 a}$}

Clinicians should inquire about medication AEs with people with MS who are taking a DMT and attempt to manage these AEs, as appropriate (Level B).

\section{Statement $\mathbf{4 b}$}

Clinicians should discuss a medication switch with people with MS for whom these AEs negatively influence adherence (Level B).

\section{Switching: Recommendation 5}

\section{Rationale}

Persistent laboratory abnormalities, such as elevated liver enzymes and decreased white blood cell counts, may prompt a discussion about switching DMT (table e-2, links.lww.com/ WNL/A376).

\section{Statement 5a}

Clinicians should monitor laboratory abnormalities found on requisite laboratory surveillance (as outlined in the medication's package insert) in people with MS who are using a DMT (Level B).

\section{Statement 5b}

Clinicians should discuss switching DMT or reducing dosage or frequency (where there are data on different doses [e.g., 
interferons, teriflunomide, azathioprine]) when there are persistent laboratory abnormalities (Level B).

\section{Switching: Recommendation 6}

\section{Rationale}

PML is a serious safety concern ${ }^{\mathrm{e} 24}$ that may affect compliance and necessitate consideration of a treatment switch. The PML risk is estimated at 4 per 1,000 overall with natalizumab ${ }^{\mathrm{e} 25}$; however, the presence and index level of JCV antibodies, longer duration use, and prior immunosuppression increase PML risk with natalizumab even further. ${ }^{\text {24 }}$ Recent updated risk estimates show that the risk of developing PML is small at antibody index values of 0.9 or less, and increases with index values greater than 1.5 in people with MS who have been treated with natalizumab for more than 2 years. ${ }^{11}$ There are rare reports of PML with the use of both fingolimod and dimethyl fumarate. ${ }^{\text {e26-e29 }}$ There are reports of PML in people with MS who are HIV-negative and using rituximab for conditions other than MS. ${ }^{\text {e30 }}$ There is a potential risk of PML with ocrelizumab use, particularly with prior immunosuppressive therapies based on its similarity to other anti-CD20 antibodies. $^{\text {e31 }}$

\section{Statement 6a}

Clinicians should counsel people with MS considering natalizumab, fingolimod, rituximab, ocrelizumab, and dimethyl fumarate about the PML risk associated with these agents (Level B).

\section{Statement 6b}

Clinicians should discuss switching to a DMT with a lower PML risk with people with MS taking natalizumab who are or become JCV antibody-positive, especially with an index of above 0.9 while on therapy (Level B).

\section{Switching: Recommendation 7}

\section{Rationale}

Immunosuppressive medications may increase the risk of opportunistic infection and malignancy, especially with prolonged use. These risks are often undefined with newer medication. Cases of cryptococcal infections have been reported with fingolimod use. ${ }^{\text {e32 }}$ Herpes family virus infections have been reported with fingolimod and natalizumab use. ${ }^{\text {e33-e35 }}$ A potential increased risk of basal cell carcinoma was recently added to the fingolimod product label. ${ }^{\text {e29 }}$

\section{Statement 7a}

Clinicians should counsel that new DMTs without long-term safety data have an undefined risk of malignancy and infection for people with MS starting or using new DMTs (Level B).

\section{Statement 7b}

If a patient with MS develops a malignancy while using a DMT, clinicians should promptly discuss switching to an alternate DMT, especially for people with MS using azathioprine, methotrexate, mycophenolate, cyclophosphamide, fingolimod, teriflunomide, alemtuzumab, or dimethyl fumarate (Level B).

\section{Statement 7c}

People with MS with serious infections potentially linked to their DMT should switch DMTs (does not pertain to PML management in people with MS using DMT) (Level B).

\section{Switching: Recommendation 8}

\section{Rationale}

Neutralizing antibodies may be produced against natalizumab and have been associated with allergic reactions. ${ }^{\text {e36,e37 }}$ These antibodies may reduce the efficacy of the medication, especially if they are persistent.

\section{Statement 8a}

Clinicians should check for natalizumab antibodies in people with MS who have infusion reactions before subsequent infusions, or in people with MS who experience breakthrough disease activity with natalizumab use (Level B).

\section{Statement $\mathbf{8 b}$}

Clinicians should switch DMTs in people with MS who have persistent natalizumab antibodies (Level B).

\section{Switching: Recommendation 9}

\section{Rationale}

People with MS taking natalizumab may discontinue natalizumab because of fear of PML risk or for pregnancy planning. Natalizumab discontinuation increases the risk of MRIdetected disease activity and MS relapse within 6 months of discontinuation, with some people with MS having an increase in disease activity above their baseline activity, referred to as rebound activity. ${ }^{\text {e38 }}$ Data are limited for assessing the appropriate choice of an alternate DMT after natalizumab discontinuation. There is evidence that initiating fingolimod 8-12 weeks after natalizumab discontinuation reduces new MRI-detected lesions compared with initiation 16 weeks after natalizumab discontinuation. Initiating fingolimod 8-12 weeks after natalizumab discontinuation increases the proportion of people with MS who are relapse free compared with initiation after 16 weeks. ${ }^{\mathrm{e} 39, \mathrm{e} 40}$ Although RCT data are unavailable, retrospective cohort data suggest that switching from natalizumab to rituximab may result in lower rates of clinical and radiologic disease activity compared with switching to fingolimod. ${ }^{\mathrm{4} 1}$

\section{Statement 9a}

Physicians must counsel people with MS considering natalizumab discontinuation that there is an increased risk of MS relapse or MRI-detected disease activity within 6 months of discontinuation (Level A).

\section{Statement 9b}

Physicians and people with MS choosing to switch from natalizumab to fingolimod should initiate treatment within 
8-12 weeks after natalizumab discontinuation (for reasons other than pregnancy or pregnancy planning) to diminish the return of disease activity (Level B).

\section{Switching: Recommendation 10}

\section{Rationale}

Relapse risk is reduced during pregnancy and increases in the postpartum period. ${ }^{\mathrm{e} 2}$ Pregnancy exposure to DMTs may pose potential risks to the fetus to varying degrees, which vary from severe malformations to no major increased risk of malformations. Risks of important early-life health outcomes such as infections, vaccination responses, asthma, and neurocognitive disorders are unknown. FDA-approved medications vary in terms of FDA recommendation for pregnancy (e.g., glatiramer acetate ["Instruct people with MS that if they are pregnant or plan to become pregnant while taking glatiramer acetate they should inform their physician"; "Women of childbearing potential should be advised to avoid becoming pregnant"] and teriflunomide ["Must be avoided during pregnancy"]). Each DMT has a separate FDA statement about pregnancy-associated risks (see individual package inserts and attached tables). Discussing these potential risks and how best to minimize them is a part of good clinical practice. The majority of human safety data for exposure to DMTs during pregnancy are derived from accidental exposure early in pregnancy. There is a paucity of safety information with second- and third-trimester exposure. ${ }^{\text {e43 }}$

\section{Statement 10a}

Clinicians should counsel women to stop their DMT before conception for planned pregnancies unless the risk of MS activity during pregnancy outweighs the risk associated with the specific DMT during pregnancy (Level B).

\section{Statement 10b}

Clinicians should discontinue DMTs during pregnancy if accidental exposure occurs, unless the risk of MS activity during pregnancy outweighs the risk associated with the specific DMT during pregnancy (Level B).

\section{Statement 10c}

Clinicians should not initiate DMTs during pregnancy unless the risk of MS activity during pregnancy outweighs the risk associated with the specific DMT during pregnancy (Level B).

\section{Stopping DMT: Recommendations}

\section{Stopping: Recommendation 1}

\section{Rationale}

No RCTs have directly addressed the question of whether, when, or why to discontinue DMTs in an individual with relapsing-remitting MS (RRMS) who has no evidence of relapses or disability progression and has stable brain imaging. The natural history of untreated RRMS is for relapses and disability accumulation to occur. Early studies suggest that most individuals with RRMS ultimately advance to secondary progressive MS (SPMS) if observed for long enough intervals, although disease course is highly variable. ${ }^{\text {e44 }}$ People with MS who are stable on DMTs may question the continued value of using DMTs. If people with MS on DMTs stop these medications, continued monitoring may show subclinical disease activity or relapse activity that would indicate a possible need for treatment resumption. In an RCT of 175 individuals taking natalizumab who had been relapse free for 1 year and had no gadolinium-enhanced lesions on MRI, participants were randomized to continue natalizumab use, switch to placebo, or switch to other therapies. Relapses occurred in $4 \%$ of those continuing natalizumab use and in 15\%-29\% of those in other treatment arms over 24 weeks. An observational study comparing outcomes in individuals who did or did not stop DMT after a period of at least 5 years without relapses found a similar risk of relapses between the groups but an increased risk of disability progression among those who stopped DMT. Younger age and lower Expanded Disability Status Scale (EDSS) scores were significant predictors of relapse (clinical or MRI) after treatment discontinuation. People with MS who are on DMTs with no evidence of ongoing disease activity may be benefiting from their DMT with disease suppression. There are no biological markers of medication efficacy that can guide decision-making in this area.

\section{Statement 1a}

In people with RRMS who are stable on DMT and want to discontinue therapy, clinicians should counsel people regarding the need for ongoing follow-up and periodic reevaluation of the decision to discontinue DMT (Level B).

\section{Statement $\mathbf{1 b}$}

Clinicians should advocate that people with MS who are stable (that is, no relapses, no disability progression, stable imaging) on DMT should continue their current DMT unless the patient and physician decide a trial off therapy is warranted (Level B).

\section{Stopping: Recommendation 2}

\section{Rationale}

People with SPMS who have relapses or active MRI-detected new lesion formation benefit from DMT. In people with SPMS who are ambulatory with or without assistance, IFN- $\beta$ reduces the risk of relapse but does not delay disability progression as measured by the EDSS, a measure that emphasizes ambulation. No RCTs have directly addressed the question of whether or when to discontinue DMTs in people with SPMS. Clinical trials have not evaluated the benefits of DMT in individuals with SPMS who are nonambulatory with respect to other clinically relevant domains, including vision, cognition, and upper limb function. Relapses are associated with more rapid disability progression in SPMS but tend to occur in those at younger ages (younger than 55 years) and earlier in the disease course. ${ }^{\mathrm{e} 45, \mathrm{e} 46}$ Among individuals with SPMS (those with and those without clinical relapses) for at least 2 
years at the time of treatment withdrawal, an EDSS of 6 or greater was associated with a $50 \%$ lower risk of relapses or MRI-detected activity after treatment discontinuation. The benefits of therapy should outweigh the risks. The use of ineffective therapy may pose harms to the affected individual, society, and the health system.

\section{Statement 2a}

Clinicians should assess the likelihood of future relapse in individuals with SPMS by assessing patient age, disease duration, relapse history, and MRI-detected activity (e.g., frequency, severity, time since most recent relapse or gadolinium-enhanced lesion) (Level B).

\section{Statement 2b}

Clinicians may advise discontinuation of DMT in people with SPMS who do not have ongoing relapses (or gadoliniumenhanced lesions on MRI activity) and have not been ambulatory (EDSS 7 or greater) for at least 2 years (Level C).

\section{Stopping: Recommendation 3}

\section{Rationale}

DMTs tested in people with CIS delay progression to MS onset. However, some people with CIS may not develop MS. e47 Risks of active relapsing disease activity are higher in younger people with CIS. ${ }^{17, \mathrm{e} 48, \mathrm{e} 49}$ In the absence of disease activity, people with CIS who are on DMTs may question the value of continuing DMTs indefinitely. There remains a gap in knowledge about stopping DMTs in people with CIS. Discussing the risks of continuing DMTs vs the risks of their use being unnecessary as part of ongoing treatment is a part of good clinical practice.

\section{Statement 3}

Clinicians should review the associated risks of continuing DMTs vs those of stopping DMTs in people with CIS using DMTs who have not been diagnosed with MS (Level B).

\section{Clinical context for all evidence}

This practice guideline reflects the complexity of decisionmaking when considering initiating, switching, or stopping DMT use for MS. The guideline panel has striven to reflect a patient-centric approach incorporating assessment of attitudes, readiness to start or change DMTs, therapy adherence, patient-specific factors (e.g., comorbidities), and an ongoing discussion of DMT use in people with MS on DMTs. The panel reviewed both FDA-approved DMTs and medications that have been used off-label for which efficacy data may be analyzed. The panel engaged in a transparent process, including extensive public review of the initial protocol, questions considered in the systematic review, and an early version of the systematic review and recommendations.

No guideline of this complexity will satisfy all audiences. The panel recognizes that the field of MS treatment is rapidly changing and the recommendations presented here may require reanalysis in light of new directions in the field and new evidence pertaining to DMT use. Issues with generalizability of randomized trials to heterogeneous real-world populations and extrapolation of short-term outcomes limit some of the conclusions. The panel anticipates needing to update this guideline in the not-too-distant future.

\section{Suggestions for future research}

Future research carried out on the following suggested topics would greatly improve informed decision-making for DMT use for MS.

High-quality evidence is needed concerning the effect of DMT for MS on outcomes deemed important by clinicians and people with MS beyond standard trials outcomes.

DMT for MS comparative efficacy studies are needed with transparent reporting in different MS subpopulations.

Clinical trials are needed to evaluate DMT benefit in individuals with SPMS who are nonambulatory with respect to other clinically relevant domains.

Studies are needed to examine whether initial high-potency treatment early in the disease course, compared with other DMTs, improves long-term outcomes.

There is a need for studies comparing highly active DMTs for MS treatment and different DMTs for CIS treatment.

Studies are warranted to determine whether switching DMTs vs continuing a DMT, despite continued disease activity, results in improved long-term outcomes.

Continued research is urged to identify biomarkers that can predict DMT efficacy in different patient subpopulations.

More research is needed to minimize risk to the pregnant woman and her fetus. Particular concerns include determining when DMT for MS should be stopped before conception, whether some agents are safer than others, and which agents might be safe enough to continue through conception and pregnancy in people with MS with active disease. More data are needed examining the risk of return of disease activity during pregnancy or the postpartum period on the mother's long-term disability risk and quality of life with DMT discontinuation during preconception, early pregnancy, or lactation.

More studies are needed to inform decisions about the possibility of DMT discontinuation, and in which circumstance, if any, discontinuation poses little or no harm.

Trial designs such as pragmatic clinical trials in clinical populations are needed to address treatment variation. 


\section{Author contributions}

Dr. Rae-Grant: study concept and design, acquisition of data, analysis or interpretation of data, drafting/revising the manuscript, critical revision of the manuscript for important intellectual content, study supervision. Dr. Day: study concept and design, analysis or interpretation of data, drafting/revising the manuscript, critical revision of the manuscript for important intellectual content. Dr. Marrie: study concept and design, acquisition of data, analysis or interpretation of data, drafting/revising the manuscript, critical revision of the manuscript for important intellectual content. Dr. Rabinstein: analysis or interpretation of data, drafting/revising the manuscript. Dr. Cree: study concept and design, drafting/revising the manuscript, critical revision of the manuscript for important intellectual content. Dr. Gronseth: analysis or interpretation of data, critical revision of the manuscript for important intellectual content, study supervision. Dr. Haboubi: study concept and design, analysis or interpretation of data, drafting/revising the manuscript. J. Halper: study concept and design, drafting/ revising the manuscript, critical revision of the manuscript for important intellectual content. Dr. Hosey: critical revision of the manuscript for important intellectual content. Dr. Jones: study concept and design, drafting/revising the manuscript, critical revision of the manuscript for important intellectual content. Dr. Lisak: study concept and design, drafting/revising the manuscript, critical revision of the manuscript for important intellectual content. Dr. Pelletier: study concept and design, drafting/revising the manuscript, critical revision of the manuscript for important intellectual content. Dr. Potrebic: study concept and design, acquisition of data, analysis or interpretation of data, drafting/revising the manuscript, critical revision of the manuscript for important intellectual content, study supervision. C. Sitcov: study concept and design, drafting/revising the manuscript, critical revision of the manuscript for important intellectual content. R. Sommers: drafting/revising the manuscript, critical revision of the manuscript for important intellectual content. Dr. Stachowiak: study concept and design, drafting/revising the manuscript, critical revision of the manuscript for important intellectual content. T.S.D. Getchius: study supervision. S.A. Merillat: drafting/revising the manuscript, study supervision. Dr. Pringsheim: study concept and design, acquisition of data, analysis or interpretation of data, drafting/revising the manuscript, critical revision of the manuscript for important intellectual content, study supervision.

\section{Acknowledgment}

The authors acknowledge the North American Research Committee on Multiple Sclerosis (NARCOMS) Registry for its assistance in administering an outcomes survey, the results of which were included in this practice guideline. NARCOMS is supported in part by the Consortium of Multiple Sclerosis Centers (CMSC) and the Foundation of the CMSC.

\section{Study funding}

This practice guideline was developed with financial support from the American Academy of Neurology (AAN). Authors who serve as AAN subcommittee members or as methodologists (A.R.-G., G.S.D., A.R., G.S.G., M.H., S.P., T.P.), or who are or were AAN staff members (T.S.D.G., S.A.M.), were reimbursed by the AAN for expenses related to travel to subcommittee meetings where drafts of manuscripts were reviewed. All authors on the panel were reimbursed by the AAN for expenses related to travel to 2 inperson meetings.

\section{Disclosure}

A. Rae-Grant receives royalties from 2 textbooks he has published, 1 on neurology and 1 on multiple sclerosis (MS); organizes and receives honoraria for grand rounds and neurology review courses; and is local primary investigator for a clinical trial with MedDay Pharmaceuticals, for which he receives no personal compensation. A. Rabinstein reports no disclosures relevant to the manuscript. B. Cree has received compensation for consulting from AbbVie, Biogen, EMD Serono, GeNeuro, MedImmune, Novartis, Shire, and Genzyme/Sanofi Aventis; has given expert testimony and prepared an affidavit for medical malpractice cases ( 1 or 2 per year) within his area of expertise; and has acted as consultant in a legal proceeding for Acorda and Biogen. G. Gronseth serves as associated editor (level of evidence review) for Neurology ${ }^{\circledR}$; serves on the editorial advisory board for Neurology Now; and is compensated by the American Academy of Neurology (AAN) for methodologic activities. G. Day holds stock in ANI Pharmaceuticals. M. Haboubi has received travel reimbursement and honoraria for grand rounds presentations in Madisonville, KY. J. Halper and J. Hosey report no disclosures relevant to the manuscript. D. Jones has received personal compensation for consulting from Biogen and Genzyme; has received honoraria from the Consortium of Multiple Sclerosis Centers (CMSC), the Multiple Sclerosis Association of America (MSAA), and the Pharmacy Quality Alliance; has received institutional research support from Biogen and the National MS Society (NMSS); has received salary support from the CMSC; and has received travel reimbursement from Biogen and Genzyme and from the AAN, Can Do MS, the CMSC, and the MSAA. R. Lisak served as the President of the CMSC and serves as a member of the Board of the DMC Foundation; has served on scientific advisory boards for Mallinckrodt, Syntimmune, Celegene, and Alexion; serves as chair of the adjudication committee of a clinical trial (PAREXEL); has received funding for travel from the CMSC, the GBS/CIDP Foundation International, the NMSS, and Syntimmune for travel to consultants meetings; has served as a journal editor for Clinical and Experimental Neuroimmunology and Clinical Neuropharmacology; has received publishing royalties from Willey for International Neurology: A Clinical Approach; has received honoraria from Mallinckrodt, Syntimmune, and Teva Pharmaceuticals, and from the consulting agencies AlphaSights, ClearView 
Healthcare Partners, GLC, and Insights Consulting; has served on a speakers bureau for Teva Pharmaceuticals for talks unrelated to pharmaceuticals; has received research support from Mallinckrodt for investigator-initiated wet bench studies, and from Acorda, Avanir, Biogen, Chugai, Genentech, MedImmune, Novartis, and Teva Pharmaceuticals for serving as a site investigator in multicenter trials; has given expert testimony, prepared an affidavit, and acted as witness for Teva Pharmaceuticals; and has acted as an expert on a patent case for Acorda. Wayne State University has received financial compensation from the NMSS for his salary as principal investigator for a research grant. R.A. Marrie receives research grants from nonprofit organizations, including Canadian Institutes of Health Research (CIHR), the CMSC, Crohn's and Colitis Canada, the NMSS, the Multiple Sclerosis Society of Canada, the Multiple Sclerosis Scientific Research Foundation, and Research Manitoba; and serves on the editorial board of Neurology. D. Pelletier has served on scientific advisory boards for Biogen, EMD Serono, Genzyme/Sanofi Aventis, Hoffman LaRoche, and Novartis; has received research support for Biogen, Genzyme, Hoffman LaRoche, and the National Institute of Neurologic Disorders and Stroke of the NIH; and has received honoraria for providing consulting services at scientific advisory board meetings from Biogen, EMD Serono, Genzyme/Sanofi Aventis, Hoffman LaRoche, and Novartis. S. Potrebic received an honorarium from CDI Quality Institute PLE for participation in a headache appropriate-use criteria panel for imaging; and receives travel reimbursement from the AAN for attending AAN Residency In-Service Training Examination Work Group meetings, AAN Axon Registry Committee meetings, AAN Guideline Development, Dissemination, and Implementation Subcommittee meetings, and the Guidelines International Network North America Evidence-based Guidelines Affecting Policy, Practice, and Stakeholders (E-GAPPS) conference. R. Sommers, C. Sitcov, and J. Stachowiak report no disclosures relevant to the manuscript. T. Getchius is a former AAN employee and reports no relevant disclosures. S. Merillat reports no disclosures relevant to the manuscript. T. Pringsheim has received research support from the CIHR and Shire Canada Inc. Go to Neurology.org/N for full disclosures.

\section{Disclaimer}

Clinical practice guidelines, practice advisories, systematic reviews, and other guidance published by the American Academy of Neurology and its affiliates are assessments of current scientific and clinical information provided as an educational service. The information (1) should not be considered inclusive of all proper treatments, methods of care, or as a statement of the standard of care; (2) is not continually updated and may not reflect the most recent evidence (new evidence may emerge between the time information is developed and when it is published or read); (3) addresses only the question(s) specifically identified; (4) does not mandate any particular course of medical care; and (5) is not intended to substitute for the independent professional judgment of the treating provider, as the information does not account for individual variation among people with MS. In all cases, the selected course of action should be considered by the treating provider in the context of treating the individual patient. Use of the information is voluntary. AAN provides this information on an "as is" basis, and makes no warranty, expressed or implied, regarding the information. AAN specifically disclaims any warranties of merchantability or fitness for a particular use or purpose. AAN assumes no responsibility for any injury or damage to persons or property arising out of or related to any use of this information or for any errors or omissions.

\section{Conflict of interest statement}

The American Academy of Neurology is committed to producing independent, critical, and truthful clinical practice guidelines (CPGs). Significant efforts are made to minimize the potential for conflicts of interest to influence the recommendations of this CPG. To the extent possible, the AAN keeps separate those who have a financial stake in the success or failure of the products appraised in the CPGs and the developers of the guidelines. Conflict of interest forms were obtained from all authors and reviewed by an oversight committee prior to project initiation. AAN limits the participation of authors with substantial conflicts of interest. The AAN forbids commercial participation in, or funding of, guideline projects. Drafts of the guideline have been reviewed by at least 3 AAN committees, a network of neurologists, Neurology peer reviewers, and representatives from related fields. The AAN Guideline Author Conflict of Interest Policy can be viewed at aan.com. For complete information on this process, access the 2011 AAN process manual, as amended. ${ }^{3}$

Received July 12, 2017. Accepted in final form February 15, 2018.

\section{References}

1. Goodin DS, Frohman EM, Garmany GP, et al; on behalf of the American Academy of Neurology and the MS Council for Clinical Practice Guidelines. Disease-modifying therapies in multiple sclerosis: subcommittee of the American Academy of Neurology and the MS Council for Clinical Practice Guidelines: report of the Therapeutics and Technology Assessment Subcommittee of the American Academy of Neurology and the MS Council for Clinical Practice Guidelines. Neurology 2002;58:169-178.

2. Rae-Grant A, Day GS, Marrie RA, et al; for the Guideline Development, Dissemination, and Implementation Subcommittee of the American Academy of Neurology. Comprehensive systematic review summary: disease-modifying therapies for adults with multiple sclerosis: report of the Guideline Development, Dissemination, and Implementation Subcommittee of the American Academy of Neurology. Neurology 2018;90:789-800.

3. American Academy of Neurology. Clinical Practice Guideline Process Manual, 2011 ed. [online]. St. Paul, MN: American Academy of Neurology; 2011. Available at: aan. com/policy-and-guidelines/guidelines/about-guidelines2/. Accessed March 12, 2016.

4. Schofield T, Elwyn G, Edwards A, Visser A. Shared decision making. Patient Educ Couns 2003;50:229-230.

5. Johnson J. On receiving the diagnosis of multiple sclerosis: managing the transition. Mult Scler 2003;9:82-88.

6. Ptacek JT, Eberhardt TL. Breaking bad news: a review of the literature. JAMA 1996; 276:496-502.

7. Mohr DC, Goodkin DE, Likosky W, et al. Therapeutic expectations of patients with multiple sclerosis upon initiating interferon beta-1b: relationship to adherence to treatment. Mult Scler 1996;2:222-226.

8. Henze T, Rieckmann P, Toyka KV, Multiple Sclerosis Therapy Consensus Group of the German Multiple Sclerosis Society. Symptomatic treatment of multiple sclerosis: Multiple Sclerosis Therapy Consensus Group (MSTCG) of the German Multiple Sclerosis Society. Eur Neurol 2006;56:78-105. 
9. Marrie RA, Horwitz RI. Emerging effects of comorbidities on multiple sclerosis. Lancet Neurol 2010;9:820-828.

10. Tettey P, Siejka D, Simpson S Jr, et al. Frequency of comorbidities and their association with clinical disability and relapse in multiple sclerosis. Neuroepidemiology 2016;46:106-113.

11. EMA confirms recommendations to minimise risk of brain infection PML with Tysabri [press release]. London: European Medicines Agency; 2016. Available at: ema.europa.eu/docs/en_GB/document_library/Press_release/2016/02/WC500202389. pdf. Accessed February 26, 2016.

12. Katsarava Z, Ehlken B, Limmroth V, et al; C.A.R.E. Study Group. Adherence and cost in multiple sclerosis patients treated with IM IFN beta-1a: impact of the CARE patient management program. BMC Neurol 2015;15:170.

13. Irwin DE, Cappell KA, Davis BM, Wu Y, Grinspan A, Gandhi SK. Differences in multiple sclerosis relapse rates based on patient adherence, average daily dose, and persistence with disease-modifying therapy: observations based on real-world data. Value Health 2015;18:A764.

14. Miller D, Barkhof F, Montalban X, Thompson A, Filippi M. Clinically isolated syndromes suggestive of multiple sclerosis, part 2: non-conventional MRI, recovery processes, and management. Lancet Neurol 2005;4:341-348.

15. Abou Zeid N, Bhatti MT. Acute inflammatory demyelinating optic neuritis: evidencebased visual and neurological considerations. Neurology 2008;14:207-223.

16. Optic Neuritis Study Group. Multiple sclerosis risk after optic neuritis: final optic neuritis treatment trial follow-up. Arch Neurol 2008;65:727-732.

17. Kuhle J, Disanto G, Dobson R, et al. Conversion from clinically isolated syndrome to multiple sclerosis: a large multicentre study. Mult Scler 2015;21:1013-1024.

18. Comi G, De Stefano N, Freedman MS, et al. Comparison of two dosing frequencies of subcutaneous interferon beta-1a in patients with a first clinical demyelinating event suggestive of multiple sclerosis (REFLEX): a phase 3 randomised controlled trial. Lancet Neurol 2012;11:33-41.

19. Miller A, Wolinsky JS, Kappos L, et al; TOPIC Study Group. Oral teriflunomide for patients with a first clinical episode suggestive of multiple sclerosis (TOPIC): a randomised, double-blind, placebo-controlled, phase 3 trial. Lancet Neurol 2014;13:977-986.

20. Girouard N, Soucy N. Patient considerations in the management of multiple sclerosis: development and clinical utility of oral agents. Patient Prefer Adherence 2011;5:101-108. 21. Brandes DW, Raimundo K, Agashivala N, Kim E. Implications of real-world adherence on cost-effectiveness analysis in multiple sclerosis. J Med Econ 2013;16:547-551.

22. Oleen-Burkey MA, Dor A, Castelli-Haley J, Lage MJ. The relationship between alternative medication possession ratio thresholds and outcomes: evidence from the use of glatiramer acetate. J Med Econ 2011;14:739-747.
23. Thomas NP, Curkendall S, Farr AM, Yu E, Hurley D. The impact of persistence with therapy on inpatient admissions and emergency room visits in the US among patients with multiple sclerosis. J Med Econ 2016;19:497-505.

24. Singer B, Lucas S, Kresa-Reahl K, Perrin Ross A, Blake P. Review: optimizing adherence to multiple sclerosis therapies. Int J MS Care 2008;10:113-126.

25. Treadaway K, Cutter G, Salter A, et al. Factors that influence adherence with diseasemodifying therapy in MS. J Neurol 2009;256:568-576.

26. Gilenya [package insert]. Gp. Basel, Sweden: Novartis Pharmaceuticals; 2014.

27. Ocrevus [package insert]. Cp. South San Francisco: Genentech; 2015.

28. Tysabri [package insert]. Tp. Cambridge, MA: Biogen; 2016.

29. Zinbryta [package insert]. Zp. Cambridge, MA: Biogen; 2016.

30. Bove R, Alwan S, Friedman JM, et al. Management of multiple sclerosis during pregnancy and the reproductive years: a systematic review. Obstet Gynecol 2014;124: 1157-1168.

31. Coyle PK. Management of women with multiple sclerosis through pregnancy and after childbirth. Ther Adv Neurol Disord 2016;9:198-210.

32. Leroy C, Rigot JM, Leroy M, et al. Immunosuppressive drugs and fertility. Orphanet J Rare Dis 2015;10:136

33. Cree BA. Update on reproductive safety of current and emerging disease-modifying therapies for multiple sclerosis. Mult Scler 2013;19:835-843.

34. Ellis R, Boggild M. Therapy-related acute leukaemia with mitoxantrone: what is the risk and can we minimise it? Mult Scler 2009;15:505-508.

35. Ellis R, Brown S, Boggild M. Therapy-related acute leukaemia with mitoxantrone: four years on, what is the risk and can it be limited? Mult Scler 2015;21:642-645.

36. Fleischer V, Salmen A, Kollar S, et al. Cardiotoxicity of mitoxantrone treatment in a German cohort of 639 multiple sclerosis patients. J Clin Neurol 2014;10: 289-295.

37. Le Page E, Leray E, Edan G; French Mitoxantrone Safety Group. Long-term safety profile of mitoxantrone in a French cohort of 802 multiple sclerosis patients: a 5-year prospective study. Mult Scler 2011;17:867-875.

38. Lucchinetti C, Bruck W, Parisi J, Scheithauer B, Rodriguez M, Lassmann H. Heterogeneity of multiple sclerosis lesions: implications for the pathogenesis of demyelination. Ann Neurol 2000;47:707-717.

39. Confavreux C, Vukusic S. Natural history of multiple sclerosis: a unifying concept. Brain 2006;129:606-616.

40. Hutchinson M, Kappos L, Calabresi PA, et al; AFFIRM and SENTINEL Investigators. The efficacy of natalizumab in patients with relapsing multiple sclerosis: subgroup analyses of AFFIRM and SENTINEL. J Neurol 2009;256: $405-415$.

\section{Seeking Concussion Abstracts by May 7}

Submit your concussion research for presentation in general poster sessions during the 2018 Sports Concussion Conference July 20 through 22 at the JW Marriott in Indianapolis, IN.

Visit AAN.com/view/ConcussionConference.

\section{AAN Wants to Help You with MIPS!}

MACRA and the Quality Payment Program and the 2018 Merit-based Incentive Payment System (MIPS) performance year began January 1 . The AAN is committed to your success and has tools and resources to help you successfully meet your performance goals with minimal amount of effort. Take action today and visit AAN.com/view/QPP or email your questions to macra@aan.com. 


\section{Neurology}

Practice guideline recommendations summary: Disease-modifying therapies for adults with multiple sclerosis: Report of the Guideline Development, Dissemination, and Implementation Subcommittee of the American Academy of Neurology Alexander Rae-Grant, Gregory S. Day, Ruth Ann Marrie, et al. Neurology 2018;90;777-788

DOI 10.1212/WNL.0000000000005347

\section{This information is current as of April 23, 2018}

\section{Updated Information \&} Services

Supplementary Material

\section{References}

\section{Citations}

\section{Subspecialty Collections}

Errata

Permissions \& Licensing

Reprints including high resolution figures, can be found at: http://n.neurology.org/content/90/17/777.full

Supplementary material can be found at: http://n.neurology.org/content/suppl/2018/04/23/90.17.777.DC1 http://n.neurology.org/content/suppl/2018/04/23/90.17.777.DC2

This article cites 34 articles, 2 of which you can access for free at: http://n.neurology.org/content/90/17/777.full\#ref-list-1

This article has been cited by 9 HighWire-hosted articles: http://n.neurology.org/content/90/17/777.full\#\#otherarticles

This article, along with others on similar topics, appears in the following collection(s):

Multiple sclerosis

http://n.neurology.org/cgi/collection/multiple_sclerosis

An erratum has been published regarding this article. Please see next page or: /content/92/2/112.full.pdf

Information about reproducing this article in parts (figures,tables) or in its entirety can be found online at:

http://www.neurology.org/about/about_the_journal\#permissions

Information about ordering reprints can be found online:

http://n.neurology.org/subscribers/advertise

Neurology ${ }^{\circledR}$ is the official journal of the American Academy of Neurology. Published continuously since 1951, it is now a weekly with 48 issues per year. Copyright ( 2018 American Academy of Neurology. All rights reserved. Print ISSN: 0028-3878. Online ISSN: 1526-632X.

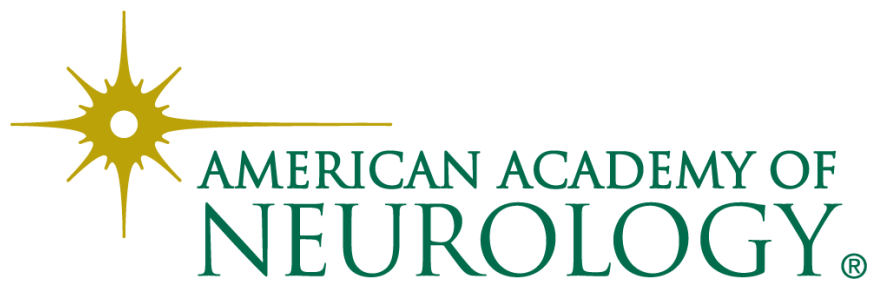




\section{Practice guideline recommendations summary: Disease-modifying} therapies for adults with multiple sclerosis: Report of the Guideline Development, Dissemination, and Implementation Subcommittee of the American Academy of Neurology

Neurology ${ }^{\circledR}$ 2019;92:112. doi:10.1212/WNL.0000000000006722

In the Special Article "Practice guideline recommendations summary: Disease-modifying therapies for adults with multiple sclerosis: Report of the Guideline Development, Dissemination, and Implementation Subcommittee of the American Academy of Neurology” by A. RaeGrant et al., ${ }^{1}$ there are errors on page 780 of the full article. The Starting: Recommendation 12 Rationale should state "With teriflunomide treatment, there may be a risk of teratogenicity from male seminal fluid, which could last for 2 years after treatment cessation if the patient is not treated with adsorption therapy" rather than "With teriflunomide treatment, there may be a risk of teratogenicity from male sperm, which could last for 2 years after treatment cessation if the patient is not treated with chelation therapy" as originally published. The authors regret the errors.

\section{Reference}

1. Rae-Grant A, Day GS, Marrie RA, et al. Practice guideline recommendations summary: disease-modifying therapies for adults with multiple sclerosis: report of the Guideline Development, Dissemination, and Implementation Subcommittee of the American Academy of Neurology. Neurology 2018;90:777-788. 